\title{
Robot-based assessment of motor and proprioceptive function identifies biomarkers for prediction of functional independence measures
}

Sayyed Mostafa Mostafavi ${ }^{1}$, Parvin Mousavi ${ }^{1}$, Sean P. Dukelow ${ }^{2}$ and Stephen H. Scott $3,4,5^{*}$

\begin{abstract}
Background: Neurological impairments following stroke impact the ability of individuals to perform daily activities, although the relative impact of individual impairments is not always clear. Recovery of sensorimotor function following stroke can vary widely, from complete recovery to modest or minimal improvements, across individuals. An important question is whether one can predict the amount of recovery based on initial examination of the individual. Robotic technologies are now being used to quantify a range of behavioral capabilities of individuals post-stroke, providing a rich set of biomarkers of sensory and motor dysfunction. The objective of the present study is to use mathematical models to identify which biomarkers best predict the ability of subjects with stroke to perform daily activities before and after rehabilitation.

Methods: The Functional Independence Measure (FIM) was quantified approximately 2 weeks and three months post-stroke in 61 ischemic and 24 hemorrhagic subjects with stroke. At 2 weeks post-stroke, subjects also completed clinical assessments and robotic assessments of sensory and motor function. A computational search algorithm, known as Fast Orthogonal Search, was used to identify the robotic and clinical biomarkers that best estimated Functional Independence Measures.
\end{abstract}

Results: Clinical and robot-based biomarkers were statistically similar at predicting FIM scores at 2 weeks ( $r=0.817$ vs. 0.774 , respectively) and 3 months ( $r=0.643$ vs. 0.685 , respectively). Importantly, robot-based biomarkers highlighted that parameters related to proprioception were influential for predicting FIM scores at 2 weeks, whereas biomarkers related to bimanual motor function were influential for predicting FIM scores at 3 months.

Conclusions: The present study provides a proof of principle on the use of robot-based biomarkers of sensory and motor dysfunction to estimate present and future FIM scores. The addition of other behavioral tasks will likely increase the accuracy of these predictions, and potentially help guide rehabilitation strategies to maximize functional recovery.

Keywords: Stroke assessment, Prognosis, Proprioception, Robotic assessment, Upper limb, Stroke

\footnotetext{
* Correspondence: steve.scott@queensu.ca

${ }^{3}$ Department of Biomedical and Molecular Sciences, Queen's University, Kingston, ON, Canada

${ }^{4}$ Providence Care, St. Mary's of the Lake Hospital, Kingston, ON, Canada

Full list of author information is available at the end of the article
}

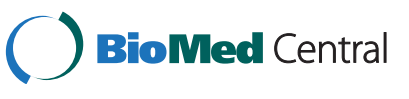

(c) 2015 Mostafavi et al. Open Access This article is distributed under the terms of the Creative Commons Attribution 4.0 International License (http://creativecommons.org/licenses/by/4.0/), which permits unrestricted use, distribution, and reproduction in any medium, provided you give appropriate credit to the original author(s) and the source, provide a link to the Creative Commons license, and indicate if changes were made. The Creative Commons Public Domain Dedication waiver (http://creativecommons.org/publicdomain/zero/1.0/) applies to the data made available in this article, unless otherwise stated. 


\section{Background}

A key focus of rehabilitation therapy following stroke is to regain the ability to perform daily activities. The Functional Independence Measure (FIM) is routinely used in Canada and the United States to score rehabilitation inpatients to quantify the ability to perform these daily activities following stroke using a 7-point scale for 13 motor and 5 cognitive tasks such as getting dressed, grooming and bowel control [1]. The FIM was designed to track changes in the functional status of patients prior to and following rehabilitation [2]. FIM scores have been shown to predict functional outcome following rehabilitation [3]. While FIM intake and discharge scores are correlated to some degree [4], predicting future abilities from early FIM scores is problematic - improved FIM scores following rehabilitation can reflect improved motor function in the affected limb (motor recovery), and/or acquired skills to perform motor actions unimanually with the unaffected limb (compensation) $[5,6]$.

The inability to perform daily activities following stroke is, of course, caused by focal damage in the brain that disrupts circuits that support various sensory, motor and cognitive processes. Several studies highlight that lesion size and location can predict motor recovery [7]. However, such predictions are not straightforward due to the close proximity of key circuits supporting various brain faculties. For example, small focal lesions near the internal capsule and thalamus can lead to varying levels of limb proprioceptive and motor impairments [8].

Another approach to predict future capabilities of daily activities is based on the underlying sensory, motor and cognitive impairments. There is an obvious link between neurological impairments and difficulties in performing daily activities. Measures of motor impairments in the affected arm generally correlate with FIM [4, 9]. As well, proprioceptive impairments also correlate with FIM scores $[9,10]$. However, improvements in FIM can occur independent of the affected limb if individuals are able to perform the activity only with their unaffected limb. This unaffected arm often also shows impairments following stroke [11], and thus also potentially impacts functional recovery.

The purpose of this study was to use robot and clinical biomarkers associated with upper limb function quantified in the first few days post-stroke to estimate the ability of subjects to perform daily activities at this same time point, as well as to predict their abilities at 3 months post-stroke. In particular, recent development of robot-based behavioural tasks provide a rich set of biomarkers of sensory and motor function, including performance of the affected and unaffected arms. Our interest was to observe which features of sensory and motor function were most important for predicting both FIM scores prior to and following rehabilitation.

\section{Methods}

\section{Participants}

Subjects with stroke were recruited after admission to St. Mary's of the Lake Hospital in Kingston, Ontario, Canada, Dr. Vernon Fanning Care Centre (Calgary, AB, Canada), and Foothills Hospital, Calgary, Alberta, Canada for clinical stroke assessment and robotic evaluation. Clinical evaluation sessions were performed within approximately two weeks post-stroke. Initial FIM measurements were also completed approximately two weeks post stroke. Evaluation of the second FIM measurements were completed 3 months $+/-1$ month post stroke. We henceforth refer to these two FIM evaluations as FIM-2w and FIM-3 $m$, respectively. Inclusion criteria were the following: (a) first clinical presentation of stroke; (b) ability to understand the task instructions; and (c) sufficient range of motion to complete robotic testing. Subjects were excluded from the study if they had a history of neurological impairments not caused by stroke, any form of acute medical illness, or musculoskeletal compromise of the shoulder or elbow. Subjects received standard rehabilitation programs that were largely based on principles of motor learning as well as neurodevelopmental techniques. Rehabilitation was generally performed each weekday for 6 to 8 weeks. The study was approved by the ethics review boards of Queen's University, University of Calgary, and Providence Care, and all subjects for the study gave their informed consent.

\section{Robotic Assessment}

Robotic assessments were performed using the KINARM exoskeleton robotic device (BKIN Technologies, Kingston, Canada, [12]). The subject's arms were abducted into the horizontal plane. The robot provided gravitational support of the limb, monitored shoulder and elbow motion, and can apply mechanical loads at the shoulder and/or elbow. A virtual reality system aligned with the plane of the arms displayed spatial targets and visual feedback of the hand, as required by each task. Participants did not use the KINARM robot except for the clinical assessments in this study. To avoid fatigue, subjects were allowed to take a break between tasks. Figure 1 depicts a schematic view of the KINARM robot for assessment of a typical subject.

\section{Visually-Guided Reaching Task}

Details of this task and its associated parameters, henceforth referred to as the Reaching task, have been described elsewhere [12]. Briefly, subjects were asked to reach "quickly and accurately" from a central target to one of eight peripheral targets located $10 \mathrm{~cm}$ away, distributed around the circumference of a circle. There was no emphasis in the task instructions on either speed or accuracy to reach to the targets so each subject selected how to trade-off these task requirements. Each trial 


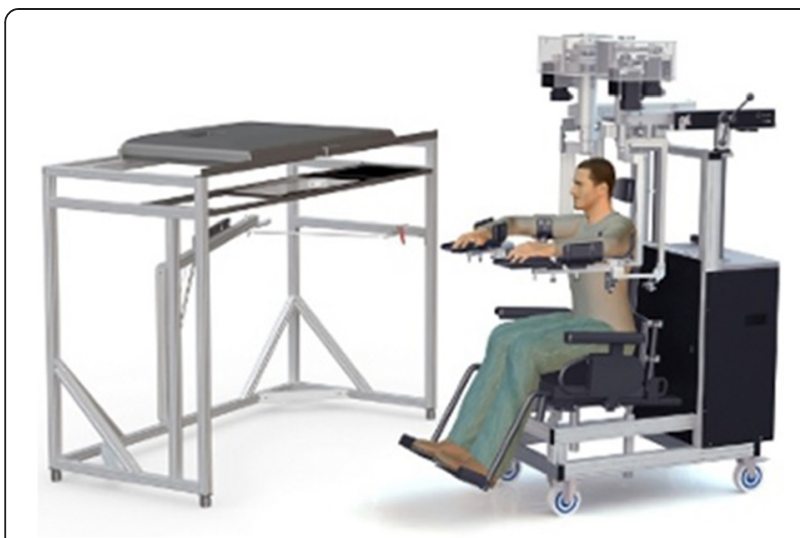

Fig. 1 KINARM exoskeleton robotic device used for evaluation of motor and proprioceptive deficits of subjects with stroke

began with subjects holding their index finger tip at a central target for 1250-1750 ms. Then a peripheral target was illuminated and subjects were given $3000 \mathrm{~ms}$ to complete the reach. Each target was presented once per block and subjects completed eight blocks for a total of 64 trials. Subjects performed this task with both their affected and unaffected arms. A total of 13 parameters were used to quantify subject performance in this task for the affected (RA) and unaffected (RU) arms (See Table 1). For each subject, the value of each measured parameter was averaged across the 64 trials. Interlimb (RI) differences in reaching performance between the two arms were also quantified.

\section{Arm-Position Matching Task}

Details of this task and its associated parameters, henceforth referred to as the Matching task, have been described elsewhere [13]. Briefly, subjects allowed the robot to passively move the affected hand (passive arm) to one of nine different spatial locations, arranged as a 3-by-3 square grid, with vision occluded [13]. When the robot stopped, subjects attempted to move the unaffected hand (active arm) to the mirror location in space. Subjects could continue to adjust the position of their active arm until they felt it was mirror-matched with the passive arm position. When subjects indicated they attained the mirror location, they informed the instructor and this was recorded as the final hand position, after which the next trial began. Target locations were such that the outer eight targets were separated by $10 \mathrm{~cm}$. The robot randomly moved from one location to the next. Subjects did not go to a standard start position between trials. Each subject completed six blocks (target locations random within a block) for a total of 54 trials. Nine parameters quantified subject performance (See Table 1).

\section{Object Hit Task}

Details of this task and its associated parameters have been described elsewhere [14]. Briefly, subjects were instructed to use virtual paddles attached to the right and left hands to hit red balls that were moving towards them on the screen. The objective of the task is to hit as many balls as possible. The balls appear on the screen from 10 different (hidden) bins, and a total of 30 balls are released from each bin in random order (all 10 bins release a ball before a bin is reused). Consequently, the game consists of a total of 300 balls falling continuously on the screen. The number of balls that appear on the screen and the speed of the ball movement increases as the task progresses, such that a single ball is moving slowly $(\sim 0.01 \mathrm{~m} / \mathrm{s})$ at the beginning and up to a maximum of 16 balls moving on the screen at $\sim 0.05 \mathrm{~m} / \mathrm{s}$ towards the end of the task. The KINARM robot provides force feedback each time a paddle hits a ball. A total of 14 parameters quantified subject performance including total balls hit, as well as spatial and motor features of the task (See Table 1).

\section{Clinical Measures of Stroke}

The Functional Independence Measure (FIM) [15], Purdue Pegboard Test [16], Modified Ashworth Score [17], and Chedoke-McMaster assessment [18] were used for clinical evaluation of subjects with stroke. These measures have established validity and reliability $[19,20]$.

The FIM measures functional abilities of subjects and is a widely used measure of disability following stroke. Good construct and validity have been established for FIM [21]. The FIM evaluates 18 areas of function, which are rated on a 1-7 scale, ranging from full dependence (1) to full independence with no required assistance (7). The 18 areas of function are categorized into 13 motor items and 5 cognitive items. We refer to the scales corresponding to the 18 items and 13 items as FIM-Total and FIM-Motor, respectively.

The Purdue Pegboard Test assesses manual dexterity and bimanual coordination [22]. The test involves two different abilities: gross movements of arms, hands, and fingers, and fine motor dexterity. The test consists of taking one peg at a time from a container and placing it into one of the holes on the board as quickly as possible with one hand. The score is expressed in the number of pegs placed in 30 seconds. In the present study, we use the sum of the scores for both hands (referred to as Purdue Combined). Poor Pegboard performance is a sign of deficits in complex, visually guided, or coordinated movements. The test is reported to have good test retest and inter-rater reliability [23, 24], but suffers from floor effects [21].

The Chedoke-McMaster Stroke Assessment Impairment Inventory is a measure used to assess physical 
Table 1 Abbreviations and description of robotic task parameters for three robotic tasks: visually guided reaching task, arm position matching task and ball drop object hit task

\begin{tabular}{|c|c|c|c|}
\hline Robotic Task & Parameters & Abbreviation & Brief Description \\
\hline \multirow[t]{13}{*}{ Visually Guided Reaching } & Reaction Time & $R A 1^{*}$ & $\begin{array}{l}\text { Time between illumination of the peripheral target and onset of } \\
\text { movement }\end{array}$ \\
\hline & First Movement Max. Speed & RA2 & Maximum hand speed during the participant's initial movement \\
\hline & First Movement Distance & RA3 & Distance the hand traveled during the participant's initial movement \\
\hline & First Movement Direction Error & RA4 & $\begin{array}{l}\text { Angular deviation between ( } a \text { ) a straight line from the hand position } \\
\text { at movement onset to the peripheral target and }(b) \text { a vector from the } \\
\text { hand position at movement onset to the hand position after the initial } \\
\text { phase of movement }\end{array}$ \\
\hline & Total Movement Time & RA5 & Total time elapsed from movement onset to offset \\
\hline & Path Length & RA6 & Total distance traveled by the hand between movement onset and offset \\
\hline & Path Length Ratio & RA7 & $\begin{array}{l}\text { Total distance traveled by the hand between movement onset and offset } \\
\text { divided by the straight line distance. }\end{array}$ \\
\hline & $\begin{array}{l}\text { Movement Time Maximum } \\
\text { Speed }\end{array}$ & RA8 & $\begin{array}{l}\text { Maximum speed that the hand traveled during the entire reaching } \\
\text { movement. }\end{array}$ \\
\hline & Number of Movement Peaks & RA9 & Number of hand speed maxima between movement onset and offset \\
\hline & Min. Max. Speed Difference & RA10 & Differences between local speed peaks and minima \\
\hline & $\begin{array}{l}\text { First Movement Maximum } \\
\text { Speed Ratio }\end{array}$ & RA11 & $\begin{array}{l}\text { Ratio of (a) the maximum hand speed during the participant's initial } \\
\text { movement to }(b) \text { the global hand speed maximum of the trial }\end{array}$ \\
\hline & First Movement Distance Ratio & RA12 & $\begin{array}{l}\text { Ratio of (a) the distance the hand traveled during the participant's initial } \\
\text { movement to (b) the distance the hand traveled between movement } \\
\text { onset and offset }\end{array}$ \\
\hline & Posture Speed & RA13 & Mean hand speed for 500 ms before peripheral target illumination \\
\hline \multirow[t]{9}{*}{ Arm Position Matching } & Variability X (m) & M1 & $\begin{array}{l}\text { Mean of standard deviation of the active hand's position over all target } \\
\text { locations in the } x \text { direction }\end{array}$ \\
\hline & Variability $Y(m)$ & M2 & $\begin{array}{l}\text { Mean of standard deviation of the active hand's position over all target } \\
\text { locations in the } y \text { direction }\end{array}$ \\
\hline & Variability XY (m) & M3 & $\begin{array}{l}\text { Mean of standard deviation of the active hand's position over all target } \\
\text { locations in the } x y \text { direction }\end{array}$ \\
\hline & Contraction/expansion ratio $\mathrm{X}$ & M4 & $\begin{array}{l}\text { Range/area of the workspace matched by the active hand relative to } \\
\text { that of the passive hand in the } x \text { direction. }\end{array}$ \\
\hline & Contraction/expansion ratio $Y$ & M5 & $\begin{array}{l}\text { Range/area of the workspace matched by the active hand relative to } \\
\text { that of the passive hand in the } y \text { direction. }\end{array}$ \\
\hline & Contraction/expansion ratio XY & M6 & $\begin{array}{l}\text { Range/area of the workspace matched by the active hand relative to } \\
\text { that of the passive hand in the } x y \text { direction. }\end{array}$ \\
\hline & Shift X (m) & M7 & $\begin{array}{l}\text { Mean of the mean error between the active and passive hands for each } \\
\text { target location over all targets in the } x \text { direction. }\end{array}$ \\
\hline & Shift $Y(m)$ & M8 & $\begin{array}{l}\text { Mean of the mean error between the active and passive hands for each } \\
\text { target location over all targets in the } y \text { direction. }\end{array}$ \\
\hline & Shift XY (m) & M9 & $\begin{array}{l}\text { Mean of the mean error between the active and passive hands for each } \\
\text { target location over all targets in the xy direction. }\end{array}$ \\
\hline \multirow[t]{7}{*}{ Object Hit } & Total hits & $\mathrm{OH} 1$ & $\begin{array}{l}\text { Number of balls that are hit and leave the display area of the subject's } \\
\text { workspace }\end{array}$ \\
\hline & Hits with Affected Arm & $\mathrm{OH} 2$ & Number of balls that are hit with the affected arm of stroke participants \\
\hline & Hits with Unaffected Arm & $\mathrm{OH} 3$ & Number of balls that are hit with the unaffected arm of stroke participants \\
\hline & Hand bias of hits & $\mathrm{OH} 4$ & $\begin{array}{l}\text { Normalized difference between the total number of hits with right and } \\
\text { left hands }\end{array}$ \\
\hline & Miss bias & $\mathrm{OH} 5$ & $\begin{array}{l}\text { Quantifies whether there was a spatial bias in position of balls missed in } \\
\text { workspace }\end{array}$ \\
\hline & Hand transition & $\mathrm{OH} 6$ & $\begin{array}{l}\text { Line in the workspace where the participant's preference switches } \\
\text { from one hand to the other }\end{array}$ \\
\hline & Hand selection overlap & $\mathrm{OH} 7$ & Quantifies whether both hands share the workspace \\
\hline
\end{tabular}


Table 1 Abbreviations and description of robotic task parameters for three robotic tasks: visually guided reaching task, arm position matching task and ball drop object hit task (Continued)

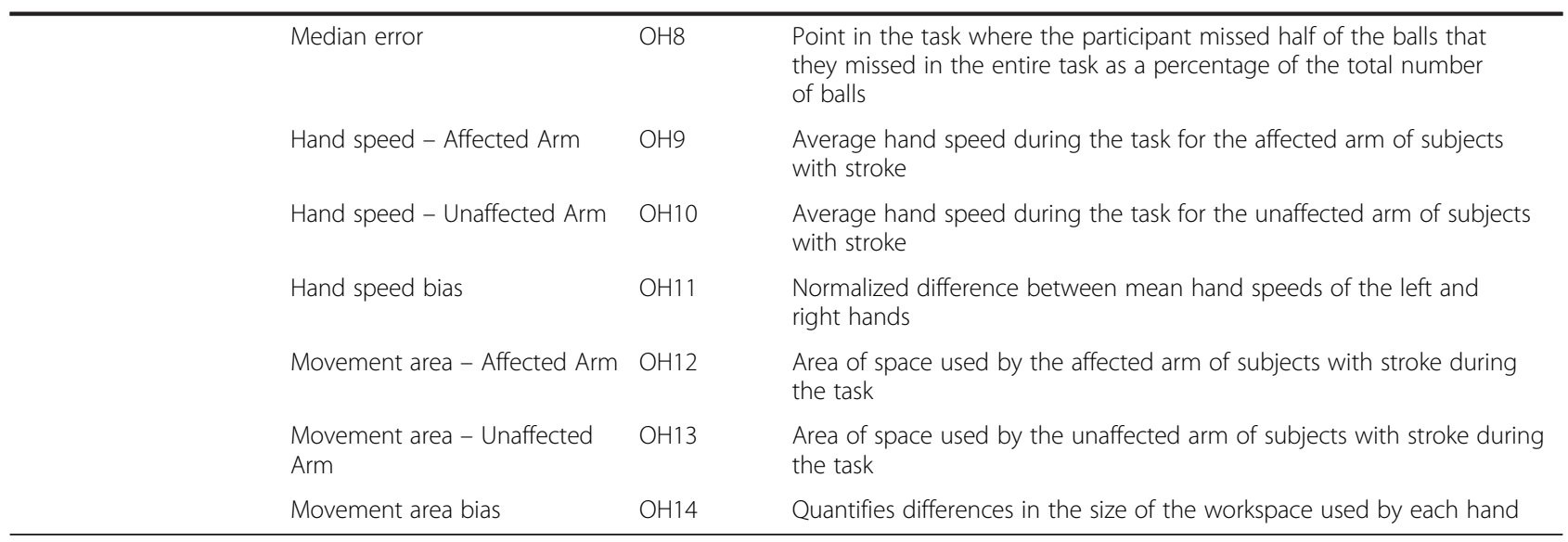

*RA followed by a number is used for abbreviation of visually guided reaching task parameter on the affected arm. RU and RI followed by a number will be used for the same parameter measured on the unaffected side and the inter-limb difference, respectively

impairment following stroke [18]. In the present study, we added the hand and arm impairment inventory scores, each evaluated on a 7-point scale [25] for both the affected and unaffected limbs (abbreviated to CMSAffected and CMS-Unaffected, respectively).

Spasticity at the elbow was assessed using the Modified Ashworth Scale [17]. It is an ordinal scale ([0, 1, 1+, $2,3,4])$, with 0 representing no spasticity. The measurements were performed for the elbow at both the affected and unaffected limbs (abbreviated to MAS-Affected and MAS-Unaffected, respectively).

The conventional subtests of the Behavioral Inattention Test (BIT) are basic pencil and paper measures to screen for visuospatial neglect [26]. A score at or below 129 is indicative of visuospatial neglect.

\section{Data analysis}

We used a normalization scheme to scale all KINARM metrics to values between 0 and 1 . The minimum $\left(\mathrm{x}_{\mathrm{min}}\right)$ and maximum $\left(\mathrm{x}_{\max }\right)$ value of each metric $(\mathrm{x})$ are used to obtain a normalized value, $(\mathrm{z}(\mathrm{x}))$, as follows:

$$
\mathrm{z}(\mathrm{x})=\frac{\mathrm{x}^{-} \mathrm{x}_{\min }}{\mathrm{x}_{\max }-\mathrm{x}_{\min }}
$$

This normalization procedure was previously found to result in more accurate classification results for stroke/ control subjects classification than a regime based on centering and scaling [27].

A system identification method known as Fast Orthogonal Search (FOS) [28] was used to identify the most informative clinical and robotic metrics that contribute towards prediction of FIM scores at approximately 2 weeks and 3 months post stroke. FOS allows for identification of a non-linear approximation consisting of terms with the highest contribution towards prediction of a desired target value [28].

In this study, the measured FIM score at approximately 2 weeks and 3 months post stroke, denoted by $S$, was used as the target value in a FOS training algorithm to form a sum of $M$ non-linear basis functions $p_{m}(n)$ with coefficients $a_{m}$ and estimate error $e(n)$ as follows

$$
S=\sum_{m=1}^{M} a_{m} \mathrm{p}_{m}(n)+e(n)
$$

The FOS training algorithm searches through all " $N$ " input candidate basis functions, where $N>>M$ and iteratively picks those candidates which contribute the largest mean square error (MSE) error reduction between the model estimate and the actual data $(S)$. This method is based on the theory of Gram-Schmidt orthogonal identification, whereby orthogonal basis functions are generated from the $p_{m}(n)$ and coefficients are found such that the MSE of the estimate is minimized [28]. The first function selected by any model using FOS is assigned a value of 1 , with a coefficient term that accounts for the constant term in the model. Following this first iteration, all subsequent basis functions are chosen from the provided pool of candidates. Non-linearity was introduced into our predicted models by including squared, cubic, sin, cosine, and logarithmic functions of the robotic metrics. A detailed description of this method is presented in [28].

FIM-related predicted measures in this study were FIM-Motor and FIM-Total at entrance to the study approximately two weeks post-stroke (FIM-Motor- $2 w$ and FIM-Total-2w) and at approximately three months poststroke (FIM-Motor-3 $\mathrm{m}$ and FIM-Total-3 $\mathrm{m}$ ). If a score 
is predicted to be outside the valid range of FIM values (e.g. $<18$ and $>126$ for FIM-Total), the predicted value is adjusted to be the minimum or maximum valid score for scores below and above the valid range, respectively.

\section{Performance Measure}

All models were evaluated by a 10 -fold cross validation procedure using $90 \%$ of the subjects' data to train the model with the remaining $10 \%$ for model testing. Overall model performance is the average of the performance of test data over the 10 folds.

To decide on the optimal number of parameters for model generalization a total of 15 models consisting of 1 to 15 basis functions were tested for each prediction. Consequently, the models that generalize best on unseen data were used as the optimal model. The addition of a higher number of basis functions results in a decrease in the models' performance on unseen validation data due to model over-fitting. The optimal number of metrics for each estimated model is reported for each model.

Performance of predicted clinical measures is reported as the $R$ value between the actual and predicted values. $R$ is a value between 0 and 1 and can be interpreted as the correlation coefficient between actual and predicted values, indicating the "goodness of fit" for such values. Moreover, the value of $R^{2}$ can be used to interpret the fraction of unexplained variance in data, since it compares the variance of the model's errors with the total data variance.

\section{Datasets for model generation}

We employed both robotic and clinical biomarkers to build predictive models. Robotic biomarkers included the Reaching task parameters recorded for the affected and unaffected arm, henceforth referred to as Reachaffected and Reach-unaffected. The set of reaching parameters describing the interlimb difference is referred to as Reach-Interlimb. A single set of data describes Matching task parameters, referred to as Match. Parameters associated with the Object Hit task are referred to as Object Hit. The last robotic dataset, All Robotic Data, contains all reaching, matching, and object hit parameters.

Clinical biomarkers are comprised of FIM-2w and a set including the Chedoke-McMaster Score, Purdue Pegboard Score, BIT Score and Modified Ashworth Score. This latter set included measures for both affected and unaffected arms, henceforth referred to as Clinicalaffected and Clinical-unaffected. Another set containing both Clinical-affected and Clinical-unaffected, henceforth referred to as Clinical-All, was also used for model generation.

For the case of each FIM prediction, all parameters in the above mentioned sets of biomarkers (i.e. robotic or clinical) were used as the candidate basis functions for the FOS training algorithm as described above. The FOS training algorithm then picks the parameters within each dataset based on their contribution towards minimizing the prediction error (as measured by MSE) for prediction of each FIM score.

\section{Results}

\section{Participant Pool}

Demographics data on the participants' age, gender, type of stroke, as well as the distribution of clinical scores is provided in Table 2. Clinical and robotic assessments were carried out on 85 patients with stroke (49 left- and 33 right-affected, 61 with ischemic and 24 with hemorrhagic stroke). The youngest participant was aged 25 , and the oldest was 82 years old, and the average age was approximately 61 years. The range of clinical scores that are covered in this study are also presented in Table 2.

\section{Estimates of FIM-2w Scores from Robotic and non- Robotic Data}

Our first analysis focused on estimation of FIM- $2 w$ scores from robotic and non-robotic biomarkers. $\mathrm{R}$ values for estimation of FIM-Motor-2w and FIM-Total$2 w$ scores are shown in Table 3. Our results show that the largest $\mathrm{R}$ values for prediction of FIM- $2 w$ scores are

Table 2 Demographics and Clinical Data

\begin{tabular}{|c|c|}
\hline Measure & Quantity \\
\hline Number of Subjects & 85 \\
\hline Male/Female & $57 / 28$ \\
\hline Affected Limb (Left-side/Right-side/Both) & $49 / 33 / 3$ \\
\hline Type of stroke (Ischemic/Hemorrhagic) & $61 / 24$ \\
\hline Age in years & $(60.6,12.5,25,82)^{a}$ \\
\hline Days since stroke to first FIM & $(8.1,4.5,1,14)^{a}$ \\
\hline Days since stroke to second FIM & $(89.6,8.9,68,125)^{a}$ \\
\hline FIM-Motor-2w score & $(66.5,21.5,13,91)^{a}$ \\
\hline FIM-Total-2w score & $(96.3,24.3,37,126)^{a}$ \\
\hline FIM-Motor-3 m score & $(85.9,10.1,27,91)^{a}$ \\
\hline FIM -Motor-3 m score & $(118.5,12.8,45,126)^{a}$ \\
\hline Purdue Pegboard Score - Combined & $(14.9,6.0,4,28.5)^{a}$ \\
\hline BIT score & $(133.8,19.1,63,146)^{a}$ \\
\hline Modified Ashworth Score - Affected Arm & $(70,5,4,6,0,0)^{b}$ \\
\hline Modified Ashworth Score - Unaffected Arm & $(83,2,0,0,0,0)^{b}$ \\
\hline Chedoke McMaster Score - Affected Arm & $(11,9,8,3,16,8,30)^{c}$ \\
\hline Chedoke McMaster Score - Affected Hand & $(14,4,5,6,16,23,16)^{c}$ \\
\hline Chedoke McMaster Score - Unaffected Arm & $(0,0,0,0,4,11,70)^{c}$ \\
\hline Chedoke McMaster Score - Unaffected Hand & $(0,0,0,0,3,24,58)^{c}$ \\
\hline
\end{tabular}


Table 3 R values for prediction of FIM-related scores at 2 weeks and 3 months post-stroke using clinical and robotic data (validation results)

\begin{tabular}{|c|c|c|c|c|c|}
\hline & & FIM-Motor-2w & FIM-Total-2w & FIM-Motor-3 m & FIM-Total-3 m \\
\hline 1 & Reach-Affected & 0.6804 & 0.7209 & 0.4205 & 0.4394 \\
\hline 2 & Reach-Unaffected & 0.3985 & 0.4045 & 0.3524 & 0.3481 \\
\hline 3 & Reach-Interlimb & 0.6324 & 0.6303 & 0.2471 & 0.2306 \\
\hline 4 & Match & 0.4608 & 0.5219 & 0.3836 & 0.2825 \\
\hline 5 & Object-Hit & 0.7237 & 0.7542 & 0.6221 & 0.5906 \\
\hline 6 & All Robotic Data & 0.7417 & 0.7745 & 0.6936 & 0.6851 \\
\hline 7 & FIM-2W & - & - & 0.6255 & 0.5987 \\
\hline 10 & Purdue-Combined & 0.7230 & 0.7393 & 0.5031 & 0.5129 \\
\hline 8 & Chedoke-Affected & 0.7310 & 0.7271 & 0.5399 & 0.5241 \\
\hline 9 & Chedoke-Unaffected & 0.3083 & 0.3338 & 0.2266 & 0.2598 \\
\hline 11 & BIT score & 0.3659 & 0.3894 & 0.3824 & 0.3808 \\
\hline 12 & Clinical-Affected & 0.8279 & 0.8106 & 0.6013 & 0.6133 \\
\hline 13 & Clinical-Unaffected & 0.5252 & 0.5310 & 0.3128 & 0.3563 \\
\hline 14 & Clinical-All & 0.8287 & 0.8172 & 0.6258 & 0.6434 \\
\hline
\end{tabular}

For dataset abbreviations (rows), see the Methods section

achieved using the Clinical-all dataset (0.829 and 0.817 for FIM-Motor-2w and FIM-Total-2w, respectively). $\mathrm{R}$ values for the robotic datasets also show high correlations of 0.742 and 0.774 for FIM-Motor-2w and FIMTotal- $2 w$, respectively. $\mathrm{R}$ values for the Object Hit dataset are almost as large as for the All Robotic Data.

Table 4 displays statistical comparisons between $\mathrm{R}$ values obtained for some of the best datasets to predict FIM-2w scores. The difference in $\mathrm{R}$ values between All Robotic Data and Clinical-All is not significant $(\mathrm{P}>0.05)$. Estimates of FIM-Motor-2w was better for the Clinical-All and the Clinical-Affected datasets than for the Object Hit dataset ( $p=0.043$ for Clinical-All and $p=0.045$ for Clinical-Affected), but not for the FIM-Total-2w.

Table 5 and 6 shows the model parameters for predictions of FIM-2w scores using All Robotic Data (top) and Clinical-All (bottom). Our models generated by FOS generalize the best using 2 basis functions for both FIMMotor-2w and FIM-Total-2w for the Clinical-All dataset. The models' performance drops on unseen validation data when including higher number of functions due to model overfitting. Basis functions are reported in Table 5 and 6 by the order in which they are selected by FOS.
Thus, the first chosen basis function is the one that contributes the most to the value of the estimated model. Repeat rates for each model parameter is generated by repetitively constructing optimal models using a randomly selected $90 \%$ subset of the original data for 100 repeats and reporting how many times each metric is picked by FOS.

For FIM-Motor-2w and FIM-Total-2w, the first dominant metric contributing to the model estimation is the Chedoke-McMaster Score-Affected, and the second dominant metric is associated with the Purdue Pegboard Score.

For predictions of FIM scores using robotic data, the best generated models generalize best using four and five parameters for FIM-Total-2w and FIM-Motor-2w, respectively. The first dominant metric contributing to the estimation of both FIM-Total-2w and FIM-Motor- $2 w$ is associated with the arm-position Matching task (M3: Variability $X Y$ ). The second and third dominant metrics are also parameters associated with arm-position Matching task (M5: Contraction/Expansion XY) and two metrics associated with the Object-Hit task (OH11: Hand speed bias, OH14: Movement Area Bias).

Table 4 P-values for comparison of $\mathrm{R}$ values between datasets

\begin{tabular}{lllllll}
\hline & Dataset 1 & Dataset 2 & FIM-Motor-2w & FIM-Total-2w & FIM-Motor-3 m & FIM-Total-3 m \\
\hline 1 & Object-Hit & FIM-2w & - & - & 0.484 & 0.468 \\
3 & Object-Hit & Clinical-All & $\mathbf{0 . 0 4 3}$ & 0.145 & 0.484 & 0.291 \\
$\mathbf{4}$ & All Robotic Data & FIM-2w & - & - & 0.203 & 0.184 \\
\hline
\end{tabular}

$\mathrm{p}$ values $<0.05$ is shown in bold 
Table 5 Robotic Metrics for Prediction of FIM-related Scores and their Corresponding Repeat Rates out of 100 Repeats for Each Model Estimation

\begin{tabular}{|c|c|c|c|c|c|c|c|c|c|c|c|c|c|}
\hline \multicolumn{14}{|c|}{ Top: predictions using robotic data } \\
\hline & $\begin{array}{l}\text { Number of model } \\
\text { parameters }\end{array}$ & $\begin{array}{l}\text { First } \\
\text { Metric }\end{array}$ & $\begin{array}{l}\text { Repeat } \\
\text { rate }(\%)\end{array}$ & $\begin{array}{l}\text { Second } \\
\text { Metric }\end{array}$ & $\begin{array}{l}\text { Repeat } \\
\text { rate }(\%)\end{array}$ & $\begin{array}{l}\text { Third } \\
\text { Metric }\end{array}$ & $\begin{array}{l}\text { Repeat } \\
\text { rate (\%) }\end{array}$ & $\begin{array}{l}\text { Fourth } \\
\text { Metric }\end{array}$ & $\begin{array}{l}\text { Repeat } \\
\text { rate }(\%)\end{array}$ & $\begin{array}{l}\text { Fifth } \\
\text { Metric }\end{array}$ & $\begin{array}{l}\text { Repeat } \\
\text { rate }(\%)\end{array}$ & $\begin{array}{l}\text { Sixth } \\
\text { Metric }\end{array}$ & $\begin{array}{l}\text { Repeat } \\
\text { rate }(\%)\end{array}$ \\
\hline \multirow[t]{3}{*}{ FIM-Motor-2w } & \multirow[t]{3}{*}{5} & M3 & 81 & $\mathrm{OH} 11$ & 26 & M5 & 45 & $\mathrm{OH} 1$ & 33 & $\mathrm{OH} 11$ & 23 & \multirow[t]{3}{*}{ N/A } & \multirow[t]{3}{*}{ N/A } \\
\hline & & RA5 & 12 & $\mathrm{OH} 12$ & 16 & $\mathrm{OH} 1$ & 24 & M5 & 21 & RA1 & 16 & & \\
\hline & & RA9 & 4 & $\mathrm{OH} 14$ & 15 & $\mathrm{OH} 12$ & 6 & $\mathrm{OH} 12$ & 8 & M9 & 10 & & \\
\hline \multirow[t]{3}{*}{ FIM-Total-2w } & \multirow[t]{3}{*}{4} & M3 & 95 & M5 & 55 & $\mathrm{OH} 14$ & 37 & M9 & 28 & \multirow[t]{3}{*}{ N/A } & \multirow[t]{3}{*}{ N/A } & \multirow[t]{3}{*}{ N/A } & \multirow[t]{3}{*}{ N/A } \\
\hline & & RA9 & 5 & $\mathrm{OH} 6$ & 17 & $\mathrm{OH} 11$ & 22 & $\mathrm{OH} 1$ & 25 & & & & \\
\hline & & & & $\mathrm{OH} 1$ & 12 & $\mathrm{OH} 12$ & 17 & M5 & 19 & & & & \\
\hline \multirow[t]{3}{*}{ FIM-Motor-3 m } & \multirow[t]{3}{*}{6} & $\mathrm{OH} 12$ & 76 & $\mathrm{OH} 13$ & 73 & RA11 & 59 & $\mathrm{OH} 5$ & 37 & RA11 & 49 & $\mathrm{OH} 5$ & 28 \\
\hline & & RA4 & 11 & RA4 & 18 & $\mathrm{OH} 13$ & 16 & RA11 & 12 & $\mathrm{OH} 5$ & 16 & RA4 & 18 \\
\hline & & $\mathrm{OH} 13$ & 5 & $\mathrm{OH} 12$ & 6 & $\mathrm{OH} 7$ & 8 & $\mathrm{OH} 7$ & 11 & RA4 & 11 & RA11 & 8 \\
\hline \multirow[t]{3}{*}{ FIM-Total-3 m } & \multirow[t]{3}{*}{5} & $\mathrm{OH} 12$ & 75 & $\mathrm{OH} 13$ & 78 & RA11 & 63 & RA7 & 34 & M9 & 27 & \multirow[t]{3}{*}{ N/A } & \multirow[t]{3}{*}{$\mathrm{N} / \mathrm{A}$} \\
\hline & & $\mathrm{OH} 11$ & 10 & RA11 & 9 & $\mathrm{OH} 5$ & 7 & $\mathrm{OH} 7$ & 12 & $\mathrm{OH} 5$ & 18 & & \\
\hline & & $\mathrm{OH} 9$ & 9 & $\mathrm{OH} 12$ & 8 & RA7 & 5 & M9 & 11 & M6 & 11 & & \\
\hline
\end{tabular}

\section{Estimates of FIM-3 m Scores from Robotic and} non-Robotic Data

We next examined the estimation of FIM-3 $m$ scores from robotic and non-robotic biomarkers. The best performance in terms of $\mathrm{R}$ values for prediction of FIM-3 m scores are achieved using the All Robotic datasets (0.694 and 0.685 for FIM-Motor-3 $m$ and FIM-Total-3 $m$, respectively). This is followed by the $\mathrm{R}$ values obtained using the Clinical-All dataset (0.626 and 0.643 for FIMMotor-3 $m$ and FIM-Total-3 $m$, respectively). Also predictions of FIM-Motor-3 $m$ and FIM-Total-3 $m$ using the respective FIM-2w scores had $\mathrm{R}$ values of 0.626 and 0.599 , respectively. Table 4 highlights that there are no statistical differences between predictions of FIM-3 m scores generated from the Clinical-All, All Robotic Data and the FIM-2w datasets.

Table 5 and 6 displays the key biomarkers that contribute to each model estimation. For FIM-Motor-3 $m$ and FIM-Total-3 $m$, the dominant first and second metrics are associated with the Object Hit task for both model estimations, while the third dominant parameter is associated with the Reaching task (affected side data). This is consistent with our results in Table 3, where $\mathrm{Ob}$ ject Hit parameters resulted in models that generalized better for prediction of FIM scores at both 2 weeks and three months post-stroke. The dominant Object Hit parameters include Movement Area-Affected Arm (OH12) and Movement Area-Unaffected Arm (OH13) for both

Table 6 Robotic Metrics for Prediction of FIM-related Scores and their Corresponding Repeat Rates out of 100 Repeats for Each Model Estimation

\begin{tabular}{|c|c|c|c|c|c|c|c|}
\hline \multicolumn{8}{|c|}{ Bottom: predictions using clinical data } \\
\hline & $\begin{array}{l}\text { Number of model } \\
\text { parameters }\end{array}$ & First Metric & Repeat rate (\%) & Second Metric & Repeat rate (\%) & Third Metric & Repeat rate (\%) \\
\hline \multirow[t]{2}{*}{ FIM-Motor-2w } & 2 & CM-A & 100 & PP & 98 & N/A & N/A \\
\hline & & & & $\mathrm{CM}-\mathrm{U}$ & 2 & & \\
\hline \multirow[t]{3}{*}{ FIM-Total-2w } & 2 & CM-A & 97 & PP & 96 & N/A & N/A \\
\hline & & PP & 3 & CM-A & 3 & & \\
\hline & & & & $\mathrm{CM}-\mathrm{U}$ & 1 & & \\
\hline \multirow[t]{3}{*}{ FIM-Motor-3 m } & 3 & PP & 100 & CM-A & 85 & PP & 75 \\
\hline & & & & $\mathrm{CM}-\mathrm{U}$ & 15 & CM-A & 16 \\
\hline & & & & & & $\mathrm{CM}-\mathrm{U}$ & 10 \\
\hline \multirow[t]{3}{*}{ FIM-Total-3 m } & 3 & PP & 100 & CM-A & 89 & CM-A & 43 \\
\hline & & & & $\mathrm{PP}$ & 7 & $\mathrm{CM}-\mathrm{U}$ & 37 \\
\hline & & & & $\mathrm{CM}-\mathrm{U}$ & 4 & PP & 20 \\
\hline
\end{tabular}


FIM-Motor-3 $m$ and FIM-Total-3 $m$ predictions. Reaching task parameters used for both predictions include First Movement Direction error (RA4), First Movement Maximum Speed Ratio (RA11), and Path Length Ratio (RA7).

Models that used clinical data for estimation of FIMMotor-3 $m$ and FIM-Total-3 $m$ scores generalize with three parameters for both scores. Similar to predictions of scores at 2 weeks post stroke, FIM-3 $m$ estimations are best predicted using a combination of Purdue Pegboard and Chedoke-McMaster assessment scores. However, for FIM-3 $m$ predictions the first dominant metric is associated with the Purdue Pegboard test for both FIM-Motor-3 $\mathrm{m}$ and FIM-Total-3 $\mathrm{m}$. The second dominant metric is associated with the Chedoke-MacMaster score, affected side. These results are consistent with our finding presented in Table 7, where both Purdue Pegboard and Chedoke MacMaster-affected side show moderate correlation values for prediction of FIM-3 $m$ scores.

\section{Estimating Clinical Scores from Robotic Data}

Our next analysis examines the relationship between robotic and clinical measures of upper limb function. Table 7 shows validation results (data not used to develop the models) using robot-based tasks to estimate the traditional clinical scores using Reaching, Matching, Object Hit, and All Robotic data. R values for prediction of Purdue-Combined and CMS-Affected using Reaching task parameters show high correlations $(0.778$ and 0.775). Predictions using All Robotic Data are slightly improved by inclusion of Object Hit and Matching data (0.791 and 0.810). Also the predictions of BIT score using robotic data shows moderate correlations with each set of robotic task data and a relatively high $\mathrm{R}$ value of 0.678 when using All Robotic Data. All R values except for the Chedoke-McMaster Score-Unaffected were significant at $P<0.005$. The correlation values for the Modified Ashworth Score are low $(\mathrm{R}=0.235$ and 0.266 for affected and unaffected arms, respectively). This is in line with the findings in [29] for the Modified Ashworth score, where they report $\mathrm{R}=0.08-0.17$ for validation data for predictions made using robot-based kinematics.

Table 7 also shows the key robotic biomarkers for prediction of these clinical scores. The dominant first and second robotic metrics for prediction of PurdueCombined and CMS-Affected are both associated with the Reaching (RA12 and RA5) and Object Hit (OH1 and $O H 8)$ task, while there is some small contribution from the Matching task as the third metric. For prediction of BIT score, the major contributions are from the Matching (M2) and Reaching task (RA11 and RA6), with

Table 7 R Values on Validation Data for Robot-Based Models to Predict Other Clinical Scores and the Robotic Metrics Associated with the Model Based on All Robotic Data

\begin{tabular}{|c|c|c|c|c|c|c|c|c|c|c|}
\hline & Reaching & Matching & Object Hit & $\begin{array}{l}\text { All Robotic } \\
\text { Data }\end{array}$ & First Metric & $\begin{array}{l}\text { Repeat } \\
\text { rate (\%) }\end{array}$ & Second Metric & $\begin{array}{l}\text { Repeat } \\
\text { rate (\%) }\end{array}$ & Third Metric & $\begin{array}{l}\text { Repeat } \\
\text { rate }(\%)\end{array}$ \\
\hline \multirow[t]{3}{*}{ Purdue-Combined } & 0.778 & 0.567 & 0.698 & 0.791 & RA12 & $61 \%$ & $\mathrm{OH} 1$ & $48 \%$ & M9 & $31 \%$ \\
\hline & & & & & $\mathrm{OH} 1$ & $28 \%$ & RA2 & $24 \%$ & $\mathrm{OH} 8$ & $21 \%$ \\
\hline & & & & & RA5 & $10 \%$ & M3 & $17 \%$ & RA3 & $12 \%$ \\
\hline \multirow[t]{3}{*}{ CMS-Affected } & 0.775 & 0.394 & 0.714 & 0.810 & RA5 & $63 \%$ & RA3 & $46 \%$ & $\mathrm{OH} 13$ & $39 \%$ \\
\hline & & & & & R12 & $21 \%$ & $\mathrm{OH} 8$ & $31 \%$ & M1 & $28 \%$ \\
\hline & & & & & RA3 & $11 \%$ & RA2 & $13 \%$ & RA2 & $19 \%$ \\
\hline \multirow[t]{3}{*}{ CMS-Unaffected } & $0.096(\mathrm{NS})$ & $0.006(N S)$ & $0.043(\mathrm{NS})$ & $0.097(\mathrm{NS})$ & $\mathrm{OH} 1$ & $18 \%$ & $\mathrm{OH} 2$ & $21 \%$ & N/A & N/A \\
\hline & & & & & M8 & $16 \%$ & M4 & $13 \%$ & & \\
\hline & & & & & M4 & $12 \%$ & RA5 & $10 \%$ & & \\
\hline \multirow[t]{3}{*}{ MAS-Affected } & 0.211 & 0.052(NS) & $0.038(\mathrm{NS})$ & 0.235 & RA1 & $45 \%$ & $\mathrm{OH} 8$ & $30 \%$ & N/A & N/A \\
\hline & & & & & $\mathrm{OH} 8$ & $21 \%$ & RA9 & $22 \%$ & & \\
\hline & & & & & $\mathrm{OH} 1$ & $13 \%$ & M8 & $10 \%$ & & \\
\hline \multirow[t]{3}{*}{ MAS-Unaffected } & 0.193(NS) & $0.063(\mathrm{NS})$ & 0.233 & 0.266 & $\mathrm{OH} 14$ & $32 \%$ & RA12 & $27 \%$ & N/A & N/A \\
\hline & & & & & $\mathrm{OH} 7$ & $24 \%$ & M1 & $19 \%$ & & \\
\hline & & & & & RA12 & $11 \%$ & RA5 & $11 \%$ & & \\
\hline \multirow[t]{3}{*}{ BIT Score } & 0.510 & 0.488 & 0.415 & 0.678 & M2 & $48 \%$ & RA6 & $38 \%$ & $\mathrm{OH} 8$ & $31 \%$ \\
\hline & & & & & RA11 & $30 \%$ & RA3 & $26 \%$ & $\mathrm{OH} 1$ & $25 \%$ \\
\hline & & & & & RA3 & $14 \%$ & M2 & $20 \%$ & M1 & $17 \%$ \\
\hline
\end{tabular}


smaller contributions from the Object Hit tasks as the third metric.

\section{Discussion}

The purpose of the present study was to identify the biomarkers of sensory and motor function of the arms at approximately 2 weeks post-stroke to estimate present and future FIM scores. Our results highlight that robotic and non-robotic assessments were not statistically different when predicting FIM-2 $w$ and FIM-3 $m$ scores. There was a general trend towards better estimates for clinical biomarkers to predict FIM-2w scores and robotic biomarkers to predict FIM-3 $m$ scores. Importantly, robot-based biomarkers of proprioceptive function were influential for predicting FIM scores at 2 weeks, whereas biomarkers related to rapid bimanual motor function were influential for predicting FIM scores at 3 months.

Robot-based measures predicted Purdue Pegboard and Chedoke-McMaster Scores with high $\mathrm{R}$ values of $\sim 0.8$ for the affected limb within approximately 2 weeks poststroke. Our predicted $\mathrm{R}$ value for the Chedoke-McMaster score is statistically higher than the results obtained in [29] for robot-based estimates of the Fugl-Meyer Assessment and Motor Power (0.427 and 0.449 respectively, on validation data). The fact that the prediction of these two clinical scores is attributed to robotic parameters associated with the Reaching and Object Hit tasks is in line with the observation that these two clinical tests are predominantly dependant on motor skills. Our improved estimates likely reflect two distinct factors. First, our mathematical models using FOS can consider non-linear relationships rather than simple linear regressions. Secondly, our range of robotic tasks covered a broader range of assessments of brain deficits including bimanual rapid visuomotor and proprioceptive tasks. While correlations were generated largely from measures of visual-guided reaching performance, there was some contribution from the limb matching and object hit tasks.

$R$ values associated with prediction of BIT scores are also relatively high ( 0.678 using all sets of robotic data). Previous studies have reported significant correlations between BIT and some robot-based metrics associated with motor function [30] (correlations as high as 0.50 ) and proprioceptive deficits [31] (correlations as high as -0.55) following stroke. In the present study we directly attempted to develop models to predict measures of spatial neglect using robot-based evaluation metrics for subjects with stroke. The correlation associated with BIT was attributed to measures of limb position matching and reaching performance with a smaller amount of contribution from the object hit task. This is in line with the inherent characteristics of reaching task, which quantifies reaching performance across targets at 8 different spatial locations and limb position matching task, which assesses sense of position across 9 different spatial targets.

Predictions of Modified Ashworth Scores resulted in poor prediction performance for both affected and unaffected limbs $(0.235$ and 0.266 , respectively). These results conform to the findings of [29] (0.171 on validation data), although our $\mathrm{R}$ values are found to be statistically significant for $P<0.005$, which may be attributed to our non-linear modeling approach.

The ability of non-robotic assessment measures to predict FIM-2w scores better than robot-based measures may be related to the impact of weakness on subject performance. Weakness is commonly observed post-stroke, and when severe, makes it impossible to perform any motor activity due to gravitational forces impeding goaldirect movements of the limb. This would directly impact the ability to perform daily activities as measured by FIM, as well as performance in the Purdue pegboard test and the Chedoke-McMaster test. In contrast, the robotic device provided weight support of the arms. Thus, a subject unable to lift their arms due to weakness may still be able to perform reaching and object hit movements to a certain degree.

It is interesting to note that the dominant robot-based biomarker to predict FIM-2w scores was related to proprioceptive function from the arm position matching task. Our previous study using this task identified high correlation between FIM scores and matching task parameters [25]. This suggests that proprioceptive function is important to plan and control many activities of daily living, such as grooming, eating and bathing. Moreover, proprioceptive deficits in the arm and leg are correlated [32], and thus, may indirectly predict difficulties in performing lower limb activities such as locomotion. Also, it has been noted previously that proprioceptive deficits do not correlate with motor skills such as reaching post stroke [9]. This explains the presence of additional nonmatching task parameters in models for predicting FIM$2 w$ scores.

Over the course of rehabilitation between first and second FIM measurements, the prediction of FIM-3 m scores using robot-based measures was slightly, although not statistically, better than clinical scores and FIM scores measured at 2 weeks. The dominant parameters attributed to the prediction of FIM-3 $m$ are associated with the object hit task. The selected parameters in the robot-based models provide some insight as to the relative success of the robot-assessment to predict future abilities to perform daily activities. First, Movement Area by both affected and unaffected hands from the object hit task were the most commonly selected parameters when developing models to predict future FIM scores. This is consistent with our understanding of the ability to perform daily activities, as the wider span of hand 
movements can prove to amount to a higher level of independence during activities of daily living. In general, dominance of object hit task parameters for prediction of future FIM scores highlights the fact that weakness in moving would amount to subjects not being able to quickly move arms for success on the object hit task. Moreover, the majority of parameters on the object task have inter-rater reliability scores above the statistically accepted norm of 0.8 [14].

Second, the relative success to predict future FIM scores may be related to the fact that robot-based models commonly selected parameters that characterize differences in limb movement, measures that compared object hit performance between the affected and unaffected limb. This includes parameters such as Miss bias, Hand speed bias and Hand selection overlap. These interlimb measures neutralize differences in each subject's motor strategy, making it easier to capture differences in motor capabilities between the limbs.

Third, the fact that the robot provided weight support for the limb may have reduced their ability to predict FIM- $2 w$ scores, but paradoxically may have improved its ability to predict FIM $3 \mathrm{~m}$ scores. Many subjects with stroke and motor deficits will improve strength with time. By alleviating weight support, the robot-based tasks assess other aspects of sensorimotor function beyond strength, allowing us to uncover other important impairments that can impact long term abilities to perform daily activities.

A recent study similar in nature to the present study was performed by Krebs, et al. [33]. They used a neural network technique to construct mathematical models for prediction of a set of clinical scores including Fugl-Meyer, National Institute of Health Stroke Scale, and Motor Power based on robotic measurements. This study was performed for a group of "completers" (who had complete data for days 7 and 90 after stroke) and "non-completers" (who had some missing data on day 7 or 90 ). The main difference between that study and the present one is that the robotic data used for model generation for that study are collected at the same time as robotic evaluation. In contrast, our study uses data collected at day 14 to make predictive models on FIM measurements approximately 3 months post stroke. Reported $\mathrm{R}^{2}$ values in that study range from $0.45-0.6$ for the "non-completer" group and 0.58-0.75 for the "completer" group. Thus, our estimates of future FIM scores are relatively good given the challenge of predicting FIM scores several months in the future and following rehabilitation therapy.

One of the limitations of our present study is the absence of behavioral tasks for assessment of cognitive brain function. FIM-Total is comprised of 5 items for assessment of cognition including memory, problem solving, and concentration. Robot-based predictions of FIM-Total-
$2 w$ tended to be better for than for FIM-Motor-2w, suggesting cognitive impairments likely impact performance in these tasks. Importantly, one of the advantages provided by robotic assessment is the potential to study behavioral tasks to quantify impairments in a broad range of sensory, motor and cognitive functions. We have recently developed several other tasks to assess sense of limb motion [10], ability to use sensory feedback for postural control [34] and bimanual motor function [35]. As well, we have developed more cognitively demanding tasks on the robotic system. An extension of the object hit task used in the present study is an object hit and avoid task, which requires additional cognitive processing to attend to the appropriate targets and inhibitory control to avoid distractors present in the workspace. Moreover, the pen-and-paper Trail Making test [36] that examines task switching has also been implemented on the robotic platform. When data on these tasks are added to the analysis, we expect that we will be able to make improved predictions on the ability of subjects to perform daily activities, as measured by the FIM, both at the present and in future time points.

One of the advantages of using robotic technologies for prediction of FIM scores lies in the ability to discover the parameters that are most selected in the models to improve the predictions of FIM. This has the potential to ultimately lead to a better understanding of the underlying changes through the rehabilitation process that may, in turn, lead to improved patient care.

Given the limited amount of available data and related studies in the use of robotic technologies for post-stroke neurorehabilitation, our collective analysis suggests that robotic technologies may enable early decision support in clinical assessment, reduce the amount of time required for assessment, and offer a reliable tool for monitoring of longitudinal changes for stroke survivors in conjunction with the current clinical scales. With addition of extra data for assessment of other areas of brain dysfunction, we expect to come up with improved decisions for patient care. The present study serves as a proof of principle, with the addition of other forms of data providing extra benefits.

\section{Conclusions}

The present study used biomarkers generated from a set of three behavioral robotic tasks to estimate measures of daily activities pre- and post-rehabilitation. Findings of the study highlight that robot-based metrics provide an accurate estimate of future FIM scores, in line with estimates provided by clinical scores at approximately 2 weeks post-stroke. While the findings of this study provide a proof of principle for use of robotic tools in clinical decision support, addition of other behavioral tasks on the robotic settings is expected to provide more accurate predictions in the future. 


\begin{abstract}
Abbreviations
FOS: fast orthogonal search; FIM: functional independence measure; FIM2W: functional independence measure assessed 2 weeks post-stroke; FIM$3 \mathrm{~m}$ : functional independence measure assessed 3 months post-stroke; CMS: chedoke mcmaster score; MAS: modified ashworth score; BIT: behavioral inattention test; KINARM: kinseological instrument for normal and altered reaching movement.
\end{abstract}

\section{Competing interests}

Dr. Scott is Co-founder and Chief Scientific Officer of BKIN technologies, the company which manufactures the KINARM robotic device. The other authors have no conflicts. The other authors have no competing interests to declare.

\section{Authors' contributions}

SMM analyzed the collected data, carried out the statistical analysis and computational modelling, and drafted the manuscript. SPD contributed to the study design, data collection and authorship of the manuscript. PM contributed to the design of the study and data analysis. SHS participated in the design of the study and robotic tasks, data analysis, and drafting of the manuscript. All authors read and approved the final manuscript.

\section{Acknowledgements}

We wish to thank Helen Bretzke, Kim Moore, Megan Metzler, Mark Piitz, Janice Yajure, and Justin Peterson for providing technical help with data preparation and processing for this study.

This work was supported by CIHR Operating grants (MOP 106662, MOP 81366 and NSP-104015), an NSERC Strategic grant (2451-06), a Heart and Stroke Foundation of Alberta, Northwest Territories and Nunavut Grant-inAid, an Ontario Research and Development - Research Excellence Award, the Hotchkiss Brain Institute, a Heart and Stroke Foundation of Alberta, Northwest Territories and Nunavut Investigatorship in Stroke Rehabilitation Research to SPD and a GSK-CIHR Chair in Neuroscience to author SHS.

\section{Author details}

${ }^{1}$ School of Computing, Queen's University, Kingston, ON, Canada. ${ }^{2}$ Hotchkiss Brain Institute, University of Calgary, Calgary, AB, Canada. ${ }^{3}$ Department of Biomedical and Molecular Sciences, Queen's University, Kingston, ON, Canada. ${ }^{4}$ Providence Care, St. Mary's of the Lake Hospital, Kingston, ON, Canada. ${ }^{5}$ Centre for Neuroscience Studies, Queen's University, Kingston K7L 3N6ON, Canada.

\section{Received: 29 May 2015 Accepted: 23 November 2015}

\section{Published online: 26 November 2015}

\section{References}

1. Keith RA, Granger CV, Hamilton BB, Sherwin FS. The functiona independence measures: a new tool for rehabilitation. Adv Clin Rehabil. 1987;1:6-18

2. Heinemann AW, Linacre JM, Hamilton BB. Prediction of rehabilitation outcomes with disability measures. Arch Phys Med Rehabil. 1994;75:133-43.

3. Chumney D, Nollinger K, Shesko K, Skop K, Spencer M, Newton RA. Ability of functional independence measure to accurately predict functional outcome of stroke-specific population: Systematic review. Journal of Rehabilitation Research \& Development. 2010;47(1):17-29.

4. Gialanella B, Santoro R. Prediction of functional outcomes in stroke patients: the role of motor patterns according to limb synergies. Aging Clin Exp Res. 2015;27(5):637-45.

5. Levin MF, Kleim JA, Wolf SL. What do motor "recovery" and "compensation" mean in patients following stroke? Neurorehabil Neural Repair. 2009;23(4):313-9.

6. Kwakkel G, Kollen B, Lindeman E. Understanding the pattern of functional recovery after stroke: facts and theories. Restor Neurol Neurosci. 2004;22(3):281-99.

7. Burke Quinlan E, Dodakian L, See J, McKenzie A, Le V, Wojnowicz M, et al. Neural function, injury, and stroke subtype predict treatment gains after stroke. Ann Neurol. 2015;77:132-45.

8. Kenzie JM, Semrau JA, Findlater SE, Herter TM, Hill MD, Scott SH, et al. Anatomical correlates of proprioceptive impairments following acute stroke: A case series. J Neurol Sci. 2014;342(1):52-61.

9. Dukelow SP, Herter TM, Bagg SD, Scott SH. The independence of deficits in position sense and visually guided reaching following stroke. J NeuroEngineering and Rehabilitation. 2012;9:72
10. Semrau JA, Herter TM, Scott SH, Dukelow SP. Robotic identification of kinesthetic deficits after stroke. Stroke. 2013:44(12):3414-21.

11. Coderre AM, Zeid AA, Dukelow SP, Demmer MJ, Moore KD, Demers MJ, et al. Assessment of upper-limb sensorimotor function of subacute stroke patients using visually-guided reaching. Neurorehabil Neural Repair. 2010;24(6):528-41.

12. Scott SH. Apparatus for measuring and perturbing shoulder and elbow joint positions and torques during reaching. J Neurosci Methods. 1999;89(2):119-27.

13. Dukelow SP, Herter TM, Moore KD, Demers MJ, Glasgow JI, Bagg SD, et al. Quantitative assessment of limb position sense following stroke. Neurorehabil Neural Repair. 2010;24(2):178-87.

14. Tyryshkin K, Coderre AM, Glasgow Jl, Herter TM, Bagg SD, Dukelow SP, et al. A robotic object hitting task to quantify sensorimotor impairments in participants with stroke. J NeuroEng Rehabil. 2014;11(1):47.

15. Ottenbacher $\mathrm{K}, \mathrm{Hsu} Y$, Granger $\mathrm{C}$, Fiedler R. The reliability of the functional independence measure: a quantitative review. Arch Phys Med Rehabil. 1996;77(12):1226-32.

16. Strauss E. A Compendium of Neuropsychological Tests: Administration, Norms, and Commentary. New York: Oxford University Press, 2006; pp. 1042. ISBN 978-0-19-515957-8.

17. Bohannon RW, Smith MB. Inter-rater reliability of a Modified Ashworth Scale of muscle spasticity. Phys Ther. 1987;67:206-7.

18. Gowland C, Stratford P, Ward M. Measuring physical impairment and disability with the Chedoke-McMaster Stroke Assessment. Stroke. 1993;24(1):58-63.

19. Kidd D, Stewart G, Baldry J, Johnson J, Rossiter D, Petruckevitch A, et al. The Functional Independence Measure: a comparative validity and reliability study. Disabil Rehabil. 1995;17(1):10-4.

20. Cohen M, Marino R. The tools of disability outcomes research functional status measures. Arch Phys Med Rehabil. 2000;81 Suppl 2:S21-9.

21. Sunderland A, Tinson D, Bradley L, Hewer RL. Arm function after stroke. an evaluation of grip strength as a measure of recovery and a prognostic indicator. J Neurol Neurosurg Psychiatry. 1989;52(11):1267-72.

22. Bode RK, Heinemann AW, Semik P, Mallinson T. Relative importance of rehabilitation therapy characteristics on functional outcomes for persons with stroke. Stroke. 2004;35(11):2537-42.

23. Radomski MV, Trombly Latham CA. Occupational Therapy for Physical Dysfunction. Lippincott Williams \& Wilkins. 2008; pp. 1140- ISBN 978-0-7817-6312-7.

24. Chen HM, Chen CC, Hsueh IP, Huang SL, Hsieh CL. Test-retest reproducibility and smallest real difference of 5 hand function tests in patients with stroke. Neurorehabil Neural Repair. 2009;23(5):435-40.

25. Brunnstrom S. Movement Therapy in Hemiplegia: A Neurolophysiological Approach. New York: Harper and Row Publishers; 1992.

26. Wilson B, Cockburn J, Halligan P. Development of a behavioral test of visuospatial neglect. Arch Phys Med Rehabil. 1987;68:98-102.

27. Chalmers N, Seaborn G, Jung JY, Glasgow JI, Scott SH. Recombination of common sensory-motor impairment evaluation techniques using a committee of classifiers. In: Annual conference of the IEEE of the engineering in medicine and biology society. Minneapolis, MN. 2009;857-60.

28. Korenberg MJ. A robust orthogonal algorithm for system identification. Biol Cybern. 1989:60:267-76.

29. Boesecker C, Dipetro L, Volpe B, Krebs HI. Kinematic robot-based evaluation scales and clinical counterparts to measure upper limb performance in patients with chronic stroke. Neurorehabil Neural Repair. 2010;24:62-9.

30. Smith DL, Akhtar AJ, Garraway WM. Proprioception and spatial neglect after stroke. Age Ageing. 1983;12:63-9.

31. Semrau JA, Wang JC, Herter TM, Scott SH, Dukelow SP. Relationship Between Visuospatial Neglect and Kinesthetic Deficits After Stroke. Neurorehabil Neural Repair. 2014;29(4):318-28.

32. Busse M, Tyson SF. How many body locations need to be tested when assessing sensation after stroke? An investigation of redundancy in the Rivermead Assessment of Somatosensory Performance. Clin Rehabil. 2009;23:91-5.

33. Krebs HI, Krams M, Agrafiotis DK, DiBernardo A, Chavez JC, Littman GS, et al. Robotic measurement of arm movements after stroke establishes biomarkers of motor recovery. Stroke. 2014:45(1):200-4.

34. Bourke TC, Coderre AM, Bagg SD, Dukelow SP, Norman KE, Scot SH. Impaired corrective responses to postural perturbations of the arm in individuals with subacute stroke. J Neuroeng Rehabil. 2015;12(1):7.

35. Lowrey CR, Jackson CPT, Bagg SD, Dukelow SP, Scott SH. A Novel Robotic Task for Assessing Impairments in Bimanual Coordination Post-Stroke. Int J Phys Med Rehabil. 2014;\$3:002.

36. Reitan, RM. Trail making test. Reitan Neuropsychology Laboratory, 1992. 